\title{
How a protein searches for its specific site on DNA: the role of intersegment transfer
}

\author{
Tao Hu and B. I. Shklovskii \\ Theoretical Physics Institute, University of Minnesota, Minneapolis, Minnesota 55455
}

(Dated: October 21, 2018)

\begin{abstract}
Proteins are known to locate their specific targets on DNA up to two orders of magnitude faster than predicted by the Smoluchowski three-dimensional diffusion rate. One of the mechanisms proposed to resolve this discrepancy is termed "intersegment transfer". Many proteins have two DNA binding sites and can transfer from one DNA segment to another without dissociation to water. We calculate the target search rate for such proteins in a dense globular DNA, taking into account intersegment transfer working in conjunction with DNA motion and protein sliding along DNA. We show that intersegment transfer plays a very important role in cases where the protein spends most of its time adsorbed on DNA.
\end{abstract}

\section{INTRODUCTION}

The description of how proteins interact with specific sites on DNA is of fundamental importance to molecular biology. The effectiveness of a DNA enzyme depends entirely on its ability to locate its target site quickly and reliably. It was recognized long ago that the search by free diffusion through three-dimensional (3D) solution is far too slow to account for the observed speed of many biological processes, and that proteins somehow arrive at their target sites up to two orders of magnitude faster than the 3D Smoluchowski rate [1, 2]

$$
J_{s}=4 \pi D_{3} b c
$$

where $b$ is the target radius and $D_{3}$ and $c$ are, respectively, the diffusion coefficient and concentration of proteins in solution. The idea to resolve this discrepancy goes back to Delbrück 3], who suggested that proteins may adsorb fairly quickly onto a nonspecific random place on DNA and then undergo one-dimensional (1D) sliding along the DNA strand, resulting in an increase of the search rate $J$ above $J_{s}$. Equivalently, we can say that the average search time for the proteins $t=1 / J$ falls below the Smoluchowski time $t_{s}=1 / J_{s}$. Below we characterize this rate enhancement by the acceleration ratio $t_{s} / t$.

The field attracted intensive attention for many years. On the theoretical front, the pioneering work by Berg, Winter and von Hippel [4] established the basis of current understanding in this field. They showed that $1 \mathrm{D}$ sliding on DNA forms a kind of "antenna" around the target site and serves to increase the effective size of the target. This large antenna size replaces the actual target size $b$ in Eq. (1), resulting in a much faster search rate. The Berg, Winter and von Hippel model predicts that the rate at which proteins find their specific target sites on DNA depends in a nonmonotonic fashion on the ionic strength of the solution, which seems to be qualitatively consistent with experiments.

In recent years, the sliding mechanism has been revisited several times [9, 10], but the question of how the protein search time depends on DNA conformation was not addressed. It is well known that DNA is coiled at length scales larger than its persistence length. When the coil cannot fit in the volume available, e.g. in the nucleoid in a prokaryotic cell, it must be a globule, as it is forced to fold back into the volume after each contact with the walls. Locally, the globule resembles a transient network with a certain mesh size (see Fig. 1). A scaling theory was recently proposed to account for the role of different DNA conformations [12]. This theory deals only with proteins with a single DNA binding site and ignores the motion of DNA in solution. Our goal in this paper is to relax these restrictions.

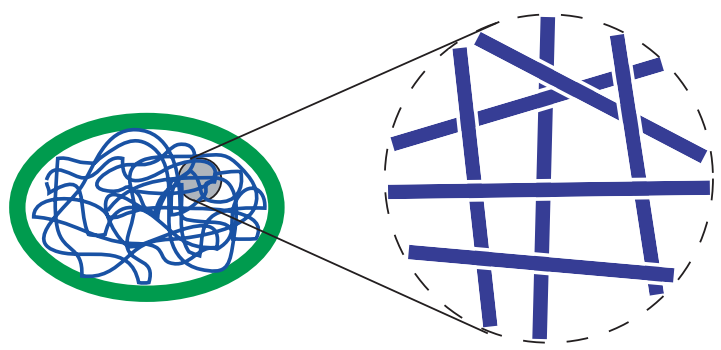

FIG. 1: A DNA globule. The long DNA is forced to return many times by the wall of a prokaryotic cell. On the right, a blown-up view shows a typical region of the transient network at a scale much smaller than the DNA persistence length $p$. This figure represents a very dense case, where the nearest neighbor distance between DNA segments, each of length $p$, is shorter than $p$.

Berg, Winter and von Hippel [4] pointed out that in addition to $1 \mathrm{D}$ sliding, proteins with two nonspecific DNA binding sites may benefit from another facilitating mechanism termed "intersegment transfer". Indeed, such proteins are capable of transiently binding to two DNA segments when the segments are close in space, even if they are well separated from each other along the DNA contour. The subsequent segmental diffusion of DNA then disrupts these double-bound states, resulting in the protein being transferred to a remote position on the DNA without net dissociation of protein into the water.

The existence of intersegment transfer in principle has been confirmed by a number of well-designed in vitro experiments [5, 6, 7]. These experiments measured the dissociation rate of proteins from a prepared complex of 
the protein and a short piece of specific DNA. The complex was placed in a solution of short nonspecific DNA molecules, and the dissociation rate was measured as a function of the concentration of nonspecific DNA. All the proteins used in [5, 6, 7], namely lac repressor [5], glucocorticoid receptor DNA-binding domain protein [6] and human Hox-D9 homeodomain [7] are believed to have two DNA binding sites so that the protein-DNA complex can adsorb a second short piece of DNA, allowing the protein to transiently form a double-bound state with two DNA pieces. This double-bound state breaks up quickly (faster than the dissociation of protein to water in the prepared protein-DNA complex) and the protein has a chance to be transferred to the newly adsorbed DNA. As a result, the dissociation rate of the complex grows linearly with the concentration of nonspecific DNA. This phenomenon of inter-DNA transfer is essentially similar to the intersegment transfer of proteins inside a single long DNA strand. Direct observation of intersegment transfer was also achieved by a scanning force microscopy study of the translocation of RNA polymerase in E. coli [8].

In this paper, we propose a scaling theory of the target search time for proteins with two DNA binding sites, which combines the effects of 3D diffusion, 1D sliding, intersegment transfer and DNA motion. Our main interest is the search time for the biologically relevant case of globular DNA. However, its complex geometrical properties combined with the several mechanisms of protein motion make this problem very complicated. Therefore, we start from a relatively simple case, namely the search time in a solution of short, straight double-helix DNA molecules among which only a small fraction carry the specific targets. In this situation we are able to include the effects of intersegment transfer and establish connections with the in vitro experiments on short DNA [5, 6, , 7]. Our analysis of this case is detailed in Sec. II, and a summary of the resulting scaling regimes is shown in Fig. 3.

In Sec. III we apply the methods developed for short DNA pieces to the case of a very dense DNA globule as shown in Fig. 1, The acceleration rate $t_{s} / t$ is shown schematically as the solid line in Fig. 2. plotted as a function of $y=\exp \left(\epsilon / k_{B} T\right)$, where $\epsilon$ is the nonspecific adsorption energy of the protein to DNA. Experimentally, the value of $y$ can be controlled through the salt concentration of the solution, since non-specific absorbtion of proteins is controlled by Coulomb interaction between negative DNA and the positive patch on the protein surface and may be screened by salt concentration. For comparison, we also plot the result of Ref. [12], which ignores DNA motion and intersegment transfer, as a dashed line. In the latter case, the acceleration rate grows first with $y$ because protein binding to DNA increases the antenna size; then the acceleration rate decays when most of the proteins are fruitlessly adsorbed far from the target (or, in other words, every protein spends most of the time adsorbed far away from the target). Finally, the acceleration rate saturates and comes to a very low plateau (not shown) when the antenna becomes as long as the DNA it- self. Hence, when DNA motion and intersegment transfer are not accounted for, there is a very strong deceleration at large ionic strength compared to the Smoluchowski rate. With the help of intersegment transfer, however, the acceleration rate saturates at a much higher level (larger than unity) because adsorbed proteins become much more effective in target search.

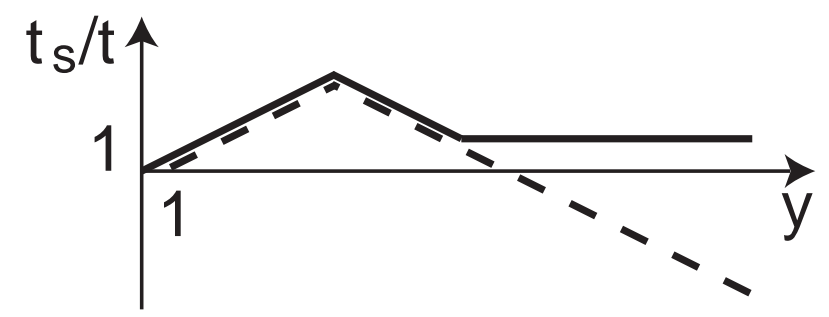

FIG. 2: Schematic dependencies of the acceleration rate on the adsorption strength $y$, with (solid line) or without (dashed line) DNA motion and intersegment transfer. Both the acceleration rate $t_{s} / t$ and $y$ are given in logarithmic scale.

In Sec. IV, we conclude with a discussion of the applicability of our model and a comparison to the previous theory [4].

\section{SIMPLE CASE: DNA IS SHORT}

\section{A. Model and approach}

We assume that within some volume $v$ a number of short, rigid (double helix) DNA molecules of length $l$ are confined, among which only one piece of DNA contains a target site of size $b$. We call this molecule the specific DNA while others are called nonspecific DNA. The system considered here is equivalent to an in vitro experiment with specific DNA concentration $1 / v$ and much larger nonspecific DNA concentration $N$.

We further assume that a protein can be adsorbed nonspecifically on DNA, and that the nonspecific adsorption energy $\epsilon$, or the corresponding constant $y=\exp \left(\epsilon / k_{B} T\right)$, is the same everywhere on the DNA and does not depend on the DNA sequence. The only exception is at the target site on the specific DNA, where the binding energy is much larger. We assume that every protein has two sites capable of binding to DNA, so that the protein can be bound to two DNA molecules at the same time.

A non-specifically bound protein is assumed to diffuse (slide) along DNA with the diffusion coefficient $D_{1}$, while protein dissolved in the surrounding water diffuses in $3 \mathrm{D}$ with diffusion coefficient $D_{3}$. In the simplest version of the theory, we assume $D_{1}=D_{3}=D$. While the protein is diffusing, the DNA molecule itself diffuses through water with diffusion coefficient $D_{t}$. Following the StokesEinstein relation, $D_{t} \sim D(b / l)$, where $b$ is the size of the protein.

The quantity of our interest is the mean time $t$ needed 
for the target site to be found by a protein. We want to look at the situation in terms of a single protein diffusing to its target. In this view, one should imagine that a protein molecule is initially introduced into a random place within volume $v$ (thus the protein concentration $c$ is $1 / v$ ), and then ask how fast the protein diffuses to its target site on the specific DNA. In order to compare the predicted time $t$ to the Smoluchowski time $t_{s}=1 / J_{s}=$ $1 / 4 \pi D c b$, we shall mainly look at the acceleration rate

$$
\frac{t_{s}}{t}=\frac{1}{t(4 \pi D c b)} \sim \frac{v}{t D b} .
$$

We note that in our scaling theory we drop away both all numerical factors and all logarithmic correction factors, which exist in the problem because it deals with strongly elongated cylinders. In this context, we will use the symbol " $\sim$ " to mean "equal up to a numerical coefficient of order one", while symbols $<$ and $>$ mean $<<$ and $>>$, respectively. Along with these simplifications, we also make several assumptions driven by pure desire to make formulae simpler and to clarify major physical ideas. We assume that all "microscopic" length scales are of the same order, namely, the target size $b$ : protein diameter, double helical DNA diameter, and the distance from DNA at which nonspecific absorbtion takes place are all considered to be roughly equal to $b$.

\section{B. Search time}

Let us imagine for a moment that there is no intersegment transfer, as is the case for a protein with only one nonspecific DNA binding site. One protein is introduced into the volume $v$. The ensuing search process for the given single protein consists of tours of $1 \mathrm{D}$ sliding along the nonspecific DNA followed by 3D diffusion in water, followed by $1 \mathrm{D}$ sliding, and so on. On its way to the target on the specific DNA, the protein will go through many adsorption and desorption cycles, and therefore the ratio of the typical time for the protein to be adsorbed, $t_{a}$, and desorbed, $t_{d}$, in a cycle should simply follow the equilibrium Boltzmann statistics:

$$
\frac{t_{a}}{t_{d}} \sim y\left(N l b^{2}\right) .
$$

The diffusion time in water per cycle $t_{d}$ can be estimated as the time a protein needs to find a DNA molecule and bind nonspecifically to it. Using Eq. (11), $t_{d} \sim 1 /(1 / v) D(N v) l \sim 1 / N D l$, where $1 / v$ stands for the protein concentration $c$ and $N v$ is the number of DNA molecules in the volume $v$. As a result $t_{a} \sim t_{d} y\left(N l b^{2}\right) \sim$ $y\left(b^{2} / D\right)$.

Let $x$ be the average length of DNA searched by the protein per cycle. Then in order to find the specific site (target) among the total $N v l / b$ sites on DNA, the protein should perform such searching cycles roughly $\mathrm{Nvl} / \mathrm{x}$ times. Therefore the search time is given by

$$
t \sim \frac{N v l}{x}\left(t_{a}+t_{d}\right)=\frac{v}{D x}\left(1+y N l b^{2}\right) .
$$

Plugging $t$ into Eq. (2), we obtain the acceleration rate

$$
\frac{t_{s}}{t} \sim \frac{1}{1+y N l b^{2}} \frac{x}{b} .
$$

We can consider two limiting cases to find expressions for $x$. At $y<l^{2} / b^{2}, x \sim\left(D t_{a}\right)^{1 / 2} \sim y^{1 / 2} b$ is just the sliding distance of the protein on one DNA molecule, while at $y>l^{2} / b^{2}, x$ is limited to the total length of DNA $l$. There are also two limiting cases for the denominator of Eq. (5). When $y$ is relatively small so that $y<1 / N l b^{2}$, i. e. the protein spends most of its time desorbed in water, the first term dominates. At $y>1 / N l b^{2}$, the protein spends most of its time adsorbed and the second term dominates. As a result we obtain four scaling regimes shown in the phase diagram of Fig. 33(a). We terminate the phase diagram at the concentration $N l^{3}=l / b$ because in a denser system liquid crystalline nematic ordering of DNA molecules becomes likely. The dependencies of the acceleration rate on $y$ for a semi-dilute solution of short DNA pieces are plotted in Fig. 4(a). The search rate is shown to increase at first due to the increase of $x$, and then decrease due to the fact that at large $y$ the protein spends most of the time adsorbed on nonspecific DNA molecules, which slows down the diffusion to the target.

Now let us move on to the case of a protein with two DNA binding sites. When a piece of DNA with an adsorbed protein collides with another DNA molecule, the protein has some probability to move directly to the new molecule. If the inter-DNA transfer is faster than the dissociation of protein into water, i.e. if the average time $\tau_{t}$ required for a protein to be transferred from one piece of DNA to another is shorter than the adsorption time $t_{a}$, then the protein can explore several DNA molecules during $t_{a}$. As a result, the protein can visit a large number of different sites during adsorption and the efficiency of $1 \mathrm{D}$ search on DNA is greatly enhanced. We find below that at large $y$ when $\tau_{t}<t_{a}$ and inter-DNA transfer dominates, the protein already spends most of the time adsorbed and $t_{a}>t_{d}$. Therefore we neglect the time spent in water $t_{d}$ and redefine $x / b$ as the number of different sites explored on the same DNA during $\tau_{t}$. The search time can then be estimated as

$$
t \sim \frac{N v l}{x} \tau_{t},
$$

so that we obtain the acceleration rate

$$
\frac{t_{s}}{t} \sim \frac{x}{N D l b \tau_{t}} .
$$

The results are shown in the diagram Fig. 3(b). At large $y$ the acceleration rate stops decreasing with $y$ and saturates. 


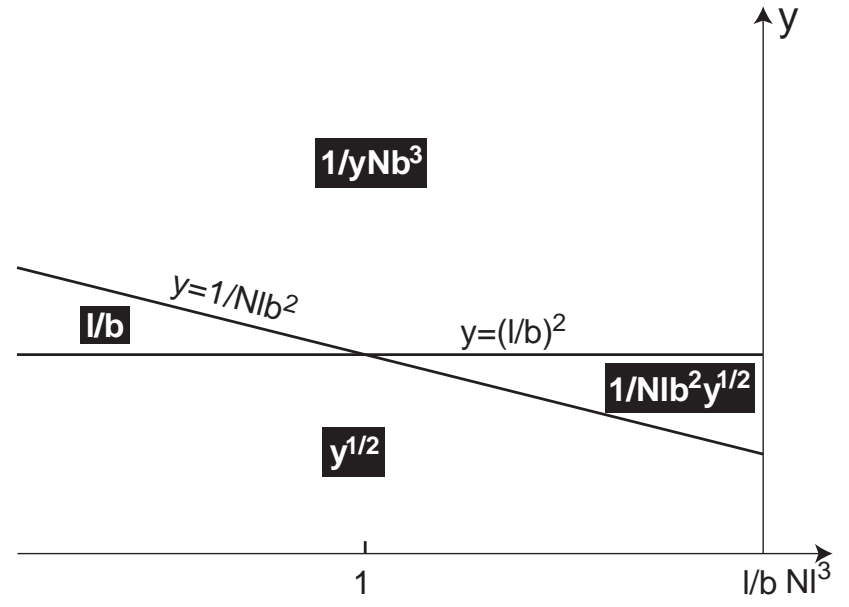

(a)

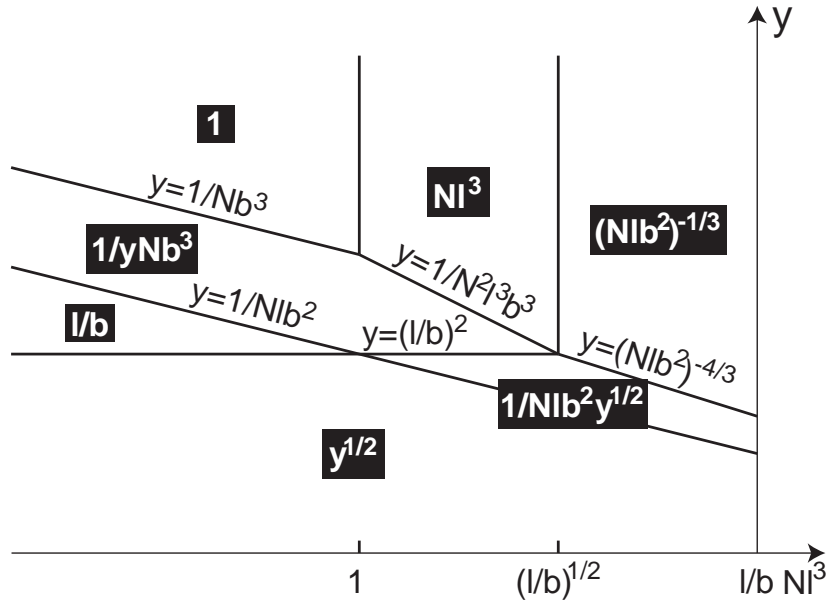

(b)

FIG. 3: "Phase diagram" for the acceleration rate $t_{s} / t$ in the plane of $y$ and $N l^{3}$, where $l$ is held constant. Both the $y$ and $N l^{3}$ axes are in the logarithmic scale. The ratio $t_{s} / t$ is shown in black on the background of each region. (a) shows scaling dependencies without inter-DNA transfer; (b) gives results with inter-DNA transfer, where $t_{s} / t$ saturates at large $y$.

We begin explaining our results by calculating the most important quantity of our theory: $\tau_{t}$. For a dilute solution of DNA molecules with $\mathrm{Nl}^{3}<1$, one can use Eq. (1) to find the time for a given DNA molecule to enter the spherical region occupied by another piece of DNA by replacing $D_{3}$ and $b$ by the DNA diffusion coefficient $D(b / l)$ and the length $l$. The result is $1 / D(b / l) N l=1 / D N b$. When the given DNA molecule enters the sphere of another molecule and diffuses over distance $l$, on average every site on the DNA has a chance to collide with the second DNA before it leaves the sphere. As a result, a protein adsorbed on one DNA can essentially always get transferred to the new one during a collision. Since in a dilute solution the diffusion time to find such a sphere containing a second DNA piece, $1 / D N b$, is larger than the diffusion time within the sphere, $l^{2} / D(b / l) \sim l^{3} / D b$, the transfer waiting time $\tau_{t}$ is the order of $1 / D N b$. Because $D \tau_{t}>l^{2}$, the protein searches $l / b$ different sites during $\tau_{t}$ and $x \sim l$. Using Eq. (6), we obtain the search time

$$
t \sim \frac{N v l}{l} \tau_{t} \sim \frac{v}{D b} \sim t_{s}
$$

When $\mathrm{Nl}^{3}>1$, the spheres containing individual DNA molecules strongly overlap. In such a semi-dilute solution, the first collision for a given DNA molecule happens when it diffuses over the nearest neighbor distance $r_{p}$. One can find $r_{p}$ by constructing an imaginary cylinder with radius $r_{p}$ around each DNA molecule, where the length of the molecule serves as the cylinder's axis. Because the excluded volume of a cylinder is $\sim l^{2} r_{p}$, the radius $r_{p}$ should satisfy $N l^{2} r_{p} \sim 1$ and thus scale as $1 / N l^{2}$. During time

$$
\tau \sim \frac{r_{p}^{2}}{D(b / l)} \sim \frac{1}{D N^{2} l^{3} b}
$$

the DNA diffuses over a distance $r_{p}$, giving every site on some segment of the DNA of length $r_{p}$ the opportunity to collide once with the nearest-neighboring DNA (see Fig. 5). After time $\tau$, the diffusing DNA and its neighbors have moved around enough that the nearest-neighboring region (shown by a dashed circle) may be considered to have shifted to a random place on the DNA.

Let us assume that the protein has just arrived at some place on the given DNA molecule. In order to be transferred to another DNA within time $\tau$, the protein must reach the segment of length $r_{p}$ (see Fig. 5) during $\tau$. Since the typical distance between the adsorbed protein and the nearest neighboring region is just proportional to the DNA length $l$, the protein will change molecules during $\tau$ when $D \tau>l^{2}$, or $N l^{3}<(l / b)^{1 / 2}$. Therefore $\tau_{t} \sim \tau$ and we obtain the search time

$$
t \sim N v \tau_{t} \sim \frac{1}{N l^{3}} \frac{v}{D b} \sim \frac{t_{s}}{N l^{3}},
$$

from which we can see that the search rate saturates $N l^{3}$ times faster than the Smoluchowski rate, and that the acceleration rate grows with DNA concentration since denser solution makes inter-DNA transfer easier.

When $N l^{3}>(l / b)^{1 / 2}$, the 1D sliding distance of protein on a single DNA molecule during $\tau$ is $x \sim(D \tau)^{1 / 2}<$ $l$. Therefore, the probability for the protein to reach the nearest neighboring region on the DNA during $\tau$ is $x / l \sim(l / b)^{1 / 2} / N l^{3}<1$. In this case the transfer waiting time $\tau_{t}>\tau$, and it should be calculated self-consistently. During $\tau_{t}$ the sliding distance $x$ of the protein is $\left(D \tau_{t}\right)^{1 / 2}$, so the probability for the protein to reach a specified nearest neighboring region is on the order of $\left(D \tau_{t}\right)^{1 / 2} / l$. Since the nearest-neighboring region changes to a random place on the DNA after $\tau$, there are $\tau_{t} / \tau$ such regions during time $\tau$. Therefore the probability for the protein to 
(a)

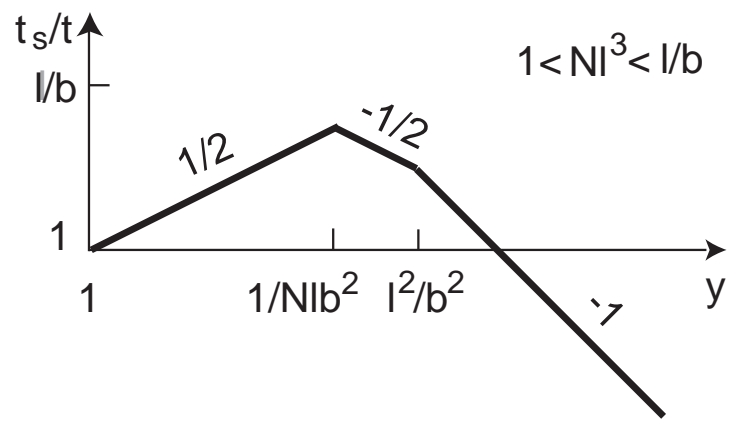

(b)

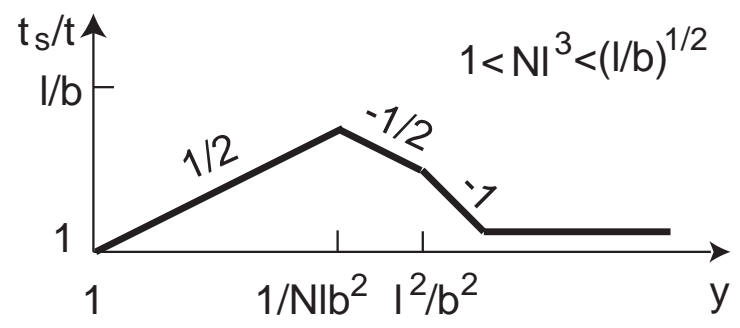

(c)

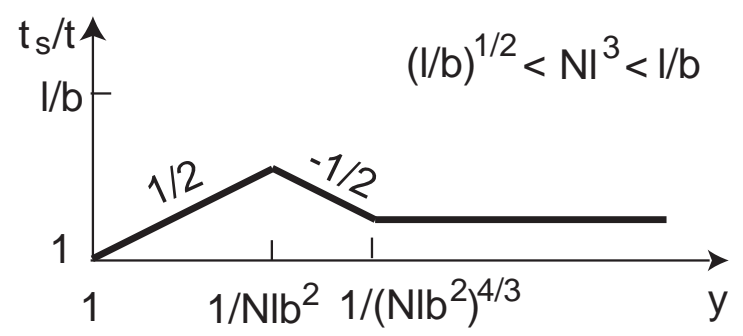

FIG. 4: Schematic dependencies of acceleration rate on $y$ for a semi-dilute $\left(\mathrm{Nl}^{3}>1\right)$ solution of short DNA pieces. (a) without inter DNA transfer; (b) and (c) with inter DNA transfer. The fraction next to each curve shows its slope (the power dependence of $t_{s} / t$ on $y$ ).

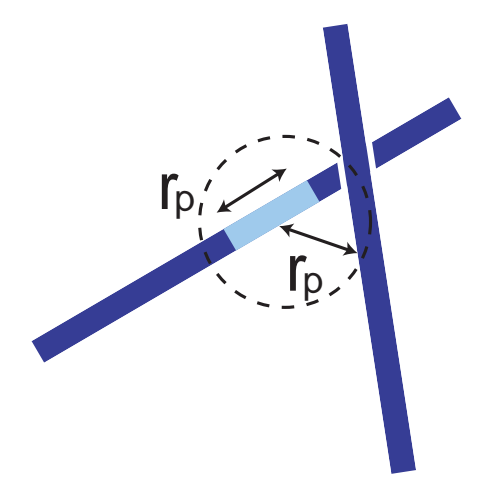

FIG. 5: Collision of a DNA molecule with its nearest neighbor at distance $r_{p}$ (other DNA molecules are not shown).

reach any one of these regions and then get transferred should satisfy

$$
\frac{\left(D \tau_{t}\right)^{1 / 2}}{l} \frac{\tau_{t}}{\tau} \sim 1
$$

As a result,

$$
\tau_{t} \sim \frac{1}{D\left(N^{2} l^{2} b\right)^{2 / 3}}
$$

and the search time is given by

$$
t \sim \frac{N v l}{\left(D \tau_{t}\right)^{1 / 2}} \tau_{t} \sim\left(N l b^{2}\right)^{1 / 3} \frac{v}{D b} \sim\left(N l b^{2}\right)^{1 / 3} t_{s} .
$$

The equations for the crossover lines at large $y$, shown in Fig. 3(b), are obtained by equating $\tau_{t}$ to $t_{a}$. This condition determines the range of parameters for which intersegment transfer takes over, i.e. when the time it takes the protein to transfer between DNA molecules is much shorter than the time the protein spends adsorbed on the DNA then we can say that intersegment transfer is the dominant mechanism. The dependencies of the acceleration rate on $y$ for semi-dilute DNA concentrations with $N l^{3}>1$ are schematically plotted in Fig. 团(b) and (c). For the purpose of comparison, we also show the dependencies for proteins with a single binding site in Fig. 团 (a).

A new feature shown by Fig. (4) (b) and (c) is that, for proteins with two DNA binding sites, inter-DNA transfer stops the search rate from decreasing and causes it to saturate at large $y$. It can be shown from equations (8), (10) and (13) that the acceleration rate is constant and $\sim 1$ when the solution is dilute and $y$ is large. The acceleration rate begins to grow as the concentration is increased past $N l^{3} \sim 1$, peaking when $N l^{3} \sim(l / b)^{1 / 2}$ and achieving a maximum value of $(l / b)^{1 / 2}$. After the peak, it decreases again and reaches $(l / b)^{1 / 3}$ when $N l^{3} \sim l / b$.

Before we move on to next section, we should emphasize that in our calculation we have completely neglected the energy barrier associated with breaking the doublebound state. We have assumed the barrier to be so small that the lifetime of the double-bound state is a small correction to the above calculated $\tau_{t}$. The search time we have found is therefore the lower limit which can be achieved with the help of intersegment transfer. In Sec. IV, we will return to this issue in more detail.

\section{Dissociation rate}

Since in experiments [5, 6, 7] the role of intersegment is inferred from measuring the dissociation rate of the prepared protein-DNA complex, in this section we calculate this rate for a protein adsorbed on a nonspecific piece of DNA dissociating to other nonspecific DNA pieces via inter-DNA transfer. 21]

The calculation is quite straightforward and the results are presented in the phase diagram of Fig. 6. The apparent dissociation rate is just $1 / t_{a}+1 / \tau_{t}$, where each term represents a possible relaxation process undergone by the adsorbed protein: either dissociation to water or intersegment transfer to another piece of DNA. The faster process dominates the rate. Since the dissociation rate 
to water decreases with the adsorption strength $y$ and the intersegment transfer rate grows with the nonspecific DNA concentration, intersegment transfer dominates the apparent dissociation rate at relatively large $y$ and $N$. We find that the enhanced dissociation rate grows linearly with nonspecific DNA concentration $N$ when the solution is dilute, in agreement with the experiments $[5,6,6]$. In semi-dilute solution, however, the dissociation rate has power law dependence on $N$, with power equal to either 2 or $4 / 3$.

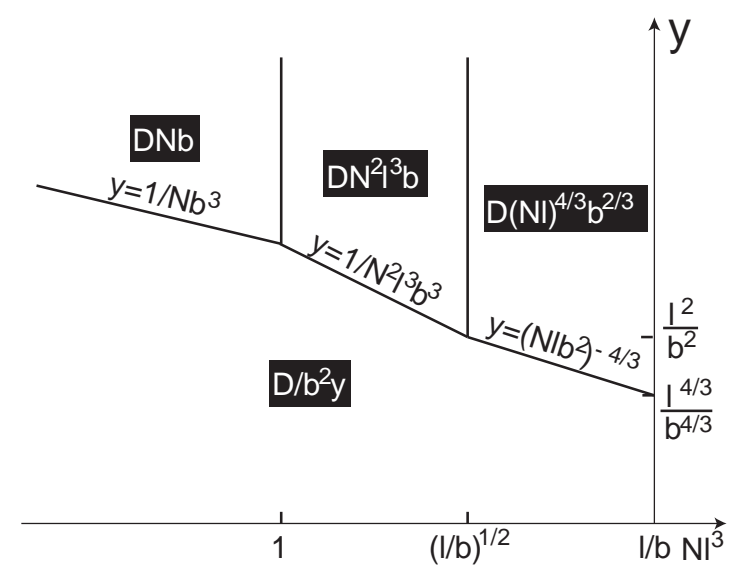

FIG. 6: "Phase diagram" for the dissociation rate under the influence of inter DNA transfer.

\section{DNA IS A GLOBULE}

After exploring the particular role of DNA motion and protein intersegment transfer for short DNA pieces, we are well prepared to generalize the above results to the more realistic case of globular DNA (see Fig. 11). We focus here on cases with large $y$, where the mechanism of intersegment transfer is important. The results for acceleration rate at small $y$, where intersegment transfer does not help much, can be found in Ref. [12].

We assume that within some volume $v$, a double helical DNA with contour length $L$ and persistence length $p \gg b$ is confined. We disregard the excluded volume of DNA, considering the DNA coil to be Gaussian and not a swollen coil, described by the Flory index $3 / 5$. This is a reasonable approximation for most realistic cases. Indeed, for many real DNA molecules such as $\lambda$-DNA, it is justified because of the large persistence length-todiameter ratio of the double helix: excluded volume in the coil remains unimportant up to DNA length about $L<p^{3} / b^{2}$ (as much as 100000 base pairs under normal ionic conditions). When the DNA is very long for a given volume, specifically, when the gaussian coil size $(L p)^{1 / 2}>v^{1 / 3}$, it cannot remain a Gaussian coil, but must fold back to make several smaller overlapping coils. In other words, it must be a globule which locally resembles a transient network.
In order to simplify our calculation, we can approximate the DNA as a series of freely-jointed straight segments (rods), each with persistence length $p$. We further restrict our study to a globule so dense that the spheres containing each rod strongly overlap (Fig. 1). Except for the connectivity, the globule is quite similar to a semidilute solution of short straight DNA pieces of length $p$ and concentration $N=(L / p) / v$ satisfying $N p^{3}>1$. In this case the diffusion distance $r_{p}$ for a given rod to experience its first collision with another rod, which may be close in space but far removed along the DNA contour, is shorter than its length $p$. As a result, one can disregard the correlation of motion between connected rods and treat the motion of each rod over the short distance $r_{p}$ separately as a normal diffusion process with diffusion coefficient $D(b / p)$.

Let us first look at a simple case where the $1 \mathrm{D}$ sliding distance $x$ for a protein on a single DNA rod is shorter than the chain length $p$. As before, we consider $x$ to be the distance traveled by the protein within a time $\tau_{t}$, the average waiting time before a protein is transferred from one DNA rod to another, uncorrelated rod. In this situation, the protein does not feel the connection between rods. Therefore, we can simply use the result for short DNA pieces, replacing the length $l$ by $p$ and using the rod concentration $N=(L / p) / v$. Then Eq. (13) gives the search time

$$
t \sim\left(N p b^{2}\right)^{1 / 3}(v / D b) \sim\left(L b^{2} / v\right)^{1 / 3} t_{s} .
$$

From Eq. (12), we find $\tau_{t} \sim 1 / D\left(N^{2} p^{2} b\right)^{2 / 3}$ and thus $x \sim\left(D \tau_{t}\right)^{1 / 2} \sim 1 /\left(N^{2} p^{2} b\right)^{1 / 3}$. So the condition $x<p$ is fulfilled when $N p^{3}>(p / b)^{1 / 2}$ or $L>\left(v / b^{2}\right)(b / p)^{3 / 2}$. Furthermore, to avoid the liquid crystalline nematic ordering of DNA chains, we assume that $N p^{3}<p / b$ or $L<v / p b$.

When the concentration of DNA rods is small enough that it falls within the range $1<N p^{3}<(p / b)^{1 / 2}$, the separation between DNA rods becomes large. Therefore, the time between collisions increases. As a result, the transfer waiting time $\tau_{t}$ grows and the $1 \mathrm{D}$ sliding distance of the protein $x$ becomes larger than $p$. In this case, one should be careful in calculating the DNA diffusion distance that results in the first collision between DNA rods. It is no longer equal to the nearest neighbor distance $r_{p} \sim 1 / N p^{2}$ between DNA rods of length $p$. To find this distance, let us concentrate on the continuous piece of length $x>p$, which spans several rods. The shortest distance from this piece of DNA to another similar piece is realized at only one of its constituent rods. The first collision that could result in transfer of the protein happens only when this particular rod diffuses over the $x$-dependent nearest neighbor distance $r(x) \sim r_{p} p / x \sim$ $1 / N p x$. During time $\tau(x) \sim r^{2}(x) / D(b / p)$, on average each DNA piece of length $x$ experiences a collision, and the protein slides a distance $x$ across the DNA. Thus, the waiting time for a protein to be transferred to another, uncorrelated DNA piece $\tau_{t} \sim \tau(x) \sim 1 / D N^{2} x^{2} p b$ should be equal to the $1 \mathrm{D}$ sliding time $x^{2} / D$ of the pro- 
tein on a single piece. This self-consistent calculation gives $x \sim(1 / N p)^{1 / 2}(p / b)^{1 / 4}$ and $\tau_{t} \sim(1 / D N p)(p / b)^{1 / 2}$. We therefore obtain the search time

$t \sim \frac{L}{x} \tau_{t} \sim\left(N p^{3}\right)^{1 / 2}\left(\frac{b}{p}\right)^{3 / 4} \frac{v}{D b} \sim\left(\frac{L b^{2}}{v}\right)^{1 / 2}\left(\frac{p}{b}\right)^{1 / 4} t_{s}$.

As explained in Ref. [12], without intersegment transfer, large values of $y$ result in the protein spending most of its time adsorbed on DNA far from the target site. The result is that the search time saturates at $L^{2} / D \sim\left(L^{2} b / v\right) t_{s}$, which is a huge deceleration compared to the Smoluchowski time. From Eqs. (14) and (15), one can easily find that at large $y$ the search time is greatly reduced below $t_{s}$ by the combination of $1 \mathrm{D}$ sliding, intersegment transfer and DNA motion. Correspondingly, the acceleration rate is enhanced and can be larger than 1, as shown by the solid line in Fig. 2. These results remain qualitatively correct for a sparser globule with $p / v^{1 / 3}<N p^{3}<1$ or $v^{2 / 3} / p<L<v / p^{2}$, where the typical mesh size of the transient network is longer than $p$ and thus the piece of DNA inside each mesh is not straight as shown in Fig. 1 but rather a small Gaussian coil. To fully account for this kind of geometry, however, one should consider a more complicated correlated segmental diffusion of DNA, and this is beyond the scope of the current paper.

Until now, we assumed that $D_{1}=D_{3}=D$, where $D_{1}$ and $D_{3}$ are the diffusion coefficients of protein on DNA and in water, respectively. In fact, the random sequence of DNA and the resulting sequence-dependent nonspecific adsorption energy most likely produces $D_{1}<$ $D_{3}$. To illustrate the role of $1 \mathrm{D}$ sliding in conjunction with intersegment transfer, we fix $D_{3}=D$ and calculate the acceleration rate for various values of $D_{1}$ following the methods explained above. The results for large $y$, where the intersegment transfer plays an important role, are shown in the plane of $D_{1} / D$ and $N p^{3}$ in Fig. 7. The dashed line corresponds to $D_{1}=D_{3}=D$. We find that the acceleration rate grows as $\left(D_{1} / D\right)^{S}$ with the index $S$ increasing from $1 / 2$ to 1 .

\section{DISCUSSION}

In our theory, we completely neglect the effect of the energy barrier $\epsilon^{*}$ associated with breaking the doublebound state and reverting to a single-bound state. Our results are therefore an upper estimate of the effect of intersegment transfer. On the other hand, a naïve guess of the barrier height is $\epsilon^{*}=\epsilon$, since to break one of the two contacts the protein has to pay the adsorption energy per binding site on one side. If this were true, the protein would be trapped in the double-bound state for the adsorption time $t_{a}$, and therefore the inter-DNA transfer could not do better job in accelerating the dissociation of the protein from the protein-DNA complex than desorption into water. As a result, adding DNA into the

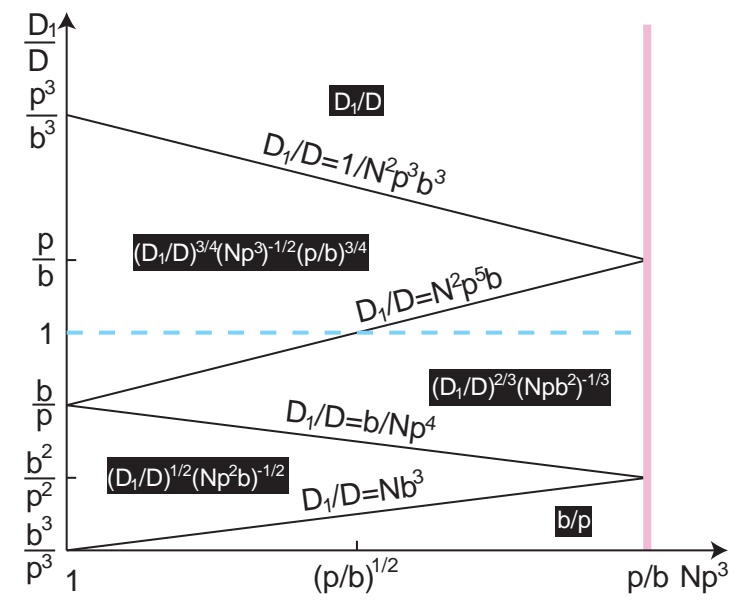

FIG. 7: "Phase diagram" for the acceleration rate at large $y$ on globular DNA, where the intersegment transfer plays an important role in the target search.

solution of a protein-DNA complex would not increase the dissociation rate of the protein, which clearly contradicts the in vitro experiments on various proteins and DNA molecules [5, 6, 7]. This suggests that in the doublebound state, the binding strength per binding site $\epsilon^{*}<\epsilon$, which could be a result of the excluded volume of close DNA molecules or the Coulomb repulsion between them.

The experiment [5] showed that the dissociation rate increases linearly with the nonspecific DNA concentration and saturates at large concentrations. This implies that at small DNA concentration, the dissociation rate is limited by the diffusion of nonspecific DNA molecules and the resulting collisions that induce inter-DNA transfer. As the DNA concentration is increased, the energy barrier for releasing the protein from the double-bound state becomes the bottleneck of the dissociation. Since the lifetime of the double-bound state does not depend on the nonspecific DNA concentration, the dissociation rate saturates. Having $\epsilon^{*}<\epsilon$ in mind, one can show that our theory is valid if the transfer waiting time $\tau_{t}$ is larger than the lifetime of the double-bound state. We can estimate this lifetime as the product of the characteristic time scale $b^{2} / D$ and the binding strength per site in the double-bound state $y^{*} \sim \exp \left(\epsilon^{*} / k_{B} T\right)$. Thus our theory works when $y^{*}\left(b^{2} / D\right)<\tau_{t}$. When $y^{*}\left(b^{2} / D\right)>\tau_{t}$, our main idea is still correct, however one should replace $\tau_{t}$ by the lifetime of the double-bound state $\left(b^{2} / D\right) y^{*}$ and repeat a similar analysis. The acceleration rate will be diminished as a result but will remain much larger than in the case without intersegment transfer.

The above discussion of $\epsilon^{*}$ assumes that the doublebound state does not affect the equilibrium Boltzmann statistics represented by Eq. (3). This places an additional restriction on $\epsilon^{*}$. The energy of the double-bound state is $2 \epsilon^{*}$. If one were to take a snapshot of the solution of short DNA pieces at a given time, the number of DNA contacts (where two DNA collide) per DNA strand is 
on the order of $N l^{2} b$, where $l^{2} b$ represents the excluded volume of a rod-like DNA. Then the limitation on $y^{*}$ can be expressed as $\exp \left(2 \epsilon^{*} / k_{B} T\right)\left(N l^{2} b\right)\left(b^{3}\right)<y l b^{2}$ or $\left(y^{*}\right)^{2}<y / N l b^{2}$.

Let us now compare our work with the treatment of intersegment transfer in Ref. [4]. While our work combines both mechanisms of 1D sliding and intersegment transfer, the Ref. [4] treats them separately. Neglecting the mechanism of protein sliding in a description of intersegment transfer results in a huge overestimation of the collision time $\tau$ and the subsequent transfer time $\tau_{t}$. Indeed one can see from Fig. 7 that if the protein cannot move on DNA, the acceleration rate is $b / p$, which is much smaller than the acceleration rate at $D_{1}=D_{3}=D$. Equivalently, neglecting intersegment transfer results in overestimation of the sliding time, sliding distance and search time. In the later review of Ref. [18], the interplay between sliding and intersegment transfer was taken into account. Qualitatively, the conclusions agree with our results, however the dependence of the intersegment transfer rate on the characteristics of DNA geometry, DNA motion, concentration and the nonspecific adsorption strength of protein to DNA $y$ was not calculated.

Finally, we note that our theory can be easily adapted to study the effective diffusion rate of a protein through a solution of polymers like DNA. This problem was studied in Ref. [17], assuming $D_{1}=0$. Following the ideas of our paper, one can expand on this study to account for the "constructive interference" of 1D sliding and intersegment transfer of protein, which was not addressed in Ref. [17]. As with target search, intersegment transfer enhances the macroscopic diffusion coefficient of proteins at large $y$, where the protein spends most of its time adsorbed on DNA. We can consider a solution of short DNA molecules, where without intersegment transfer the effective diffusion coefficient of the protein is decreased by nonspecific adsorption to DNA and eventually saturates at the DNA diffusion coefficient $D(b / l)$. In a dilute solution, intersegment transfer does not assist the macroscopic diffusion of proteins, since each DNA molecule is far removed from other molecules and therefore the macroscopic displacement of protein is determined mainly by the motion of the DNA. In a semidilute solution, however, where $1<N l^{3}<l / b, 1 \mathrm{D}$ sliding on DNA becomes important. When $1<N l^{3}<(l / b)^{1 / 2}$, $D \tau_{t}>l^{2}$ and the 1D sliding distance of protein during time $\tau_{t}$ is limited to the length of DNA $l$. In this case $\tau_{t} \sim \tau$. Using Eq. (6), the effective diffusion coefficient is obtained as $l^{2} / \tau_{t} \sim N^{2} l^{6} D(b / l)$. At higher densities when $(l / b)^{1 / 2}<N l^{3}<l / b, D \tau_{t}<l^{2}$. As a result, the nonspecific adsorption of protein on DNA does not hinder the diffusion of protein at all and the macroscopic diffusion coefficient is just $D$. For $D_{1} \neq D_{3}$, a similar analysis can be performed.

One further application of our theory is to the problem of dynamic (stirred) percolation, e.g., the conductivity of well-conducting wires in some insulating liquid. It is well known that if the wires are randomly frozen in the liquid, the conductivity vanishes below the percolation threshold [19]. However, because of the diffusion of wires in the liquid, the charge carriers are not trapped within finite clusters of wires. Instead, they can hop from one wire to another when the wires approach close to each other. This results in a finite conductivity below the percolation threshold [20]. For such systems, one can find the macroscopic diffusion coefficient of the charge carriers and then map it to the effective conductivity of the system.

\section{Acknowledgments}

We are grateful to A. Yu. Grosberg and M. G. Fried for helpful discussions. We acknowledge B. Skinner's kind help in preparing the manuscript. TH acknowledges support of the Anatoly Larkin Fellowship.
[1] A. D. Riggs, S. Bourgeois and M. Cohn, J. Mol. Bio. 53, 401 (1970).

[2] P. H. Richter and M. Eigen, Biophys. Chem. 2, 255 (1974).

[3] G. Adam, and M. Delbrück, Structural Chemistry and Molecular Biology. A. Rich, and N. Davidson, editors., pp. 198-215, (W. H. Freeman, San Francisco, CA 1968)

[4] O. G. Berg, R. B. Winter and P. H. von Hippel, Biochemistry, 20, 6929 (1981).

[5] M. G. Fried and D. M. Crothers, J. Mol. Biol. 172, 263 (1984).

[6] B. A. Lieberman and S. K. Nordeen, J. Biol. Chem. 272, 1061 (1997).

[7] J. Iwahara and G. M. Clore, J. Am. Chem. Soc. 128, 404 (2006).

[8] C. Bustamante, M. Gutholdi, X. Zhu and G. Yang, J. Biol. Chem. 274, 16665 (1999).
[9] S. E. Halford and J. F. Marko, Nucleic Acids Res. 32, 3040 (2004).

[10] M. Slutsky and L. A. Mirny, Biophys. J. 87, 4021 (2004).

[11] A. Yu. Grosberg and A. Khoklov, Statistical Physics of Macromolecules, (AIP, New York, 1994).

[12] Tao Hu, A. Yu. Grosberg and B. I. Shklovskii, Biophys. J. 90, 2731 (2006).

[13] Tao Hu, A. Yu. Grosberg and B. I. Shklovskii, Phys. Rev. B 73, 155434 (2006)

[14] R. D. Icenogle and E. L. Elson, Biopolymers 22, 1949 (1983).

[15] N. Shimamoto, J. Biol. Chem. 274, 15293 (1999).

[16] O. G. Berg, Biopolymers, 18, 2861 (1979).

[17] O. G. Berg, Biopolymers, 25, 811 (1986).

[18] O. G. Berg, in The Biology of Nonspecific DNA-Protein Interactions, edited by A. Revzin, (CRC Press, 1990) p. 71. 
[19] B. I. Shklovskii and A. L. Efros, Electronic Properties of Doped Semiconductors (Springer-Verlag, Berlin, 1984).

[20] G. S. Grest, I. Webman, S. A. Safran and A. L. R. Bug, Phys. Rev. A, 33, 2842 (1986).

[21] Our model serves as a simple generalization of experimental systems where the protein is specifically adsorbed to its target on the DNA in the complex and the DNA free in solution can be specific or nonspecific [5, 6, 7]. We argue that this generalization does not change the main feature of the problem, which is determined by the frequency at which the free DNA molecules collide with the protein-DNA complex. The difference lies in the transfer probability per collision. On the nonspecific DNA, the protein can slide freely. In contrast, protein on the spe- cific DNA spends most of the time adsorbed to its target. Thus, to experience a transfer the complex should collide with another piece of DNA exactly at the position of target, which results in smaller transfer probability. However the specifically adsorbed protein can first slide into nonspecific sites and then dissociate into the bulk solution or transfer to other DNA molecules [15]. In this way the decreased transfer probability is somewhat compensated, and it becomes closer to the case of dissociation from nonspecific DNA. More importantly, the process of target search involves the protein making direct transfers between nonspecific segments of DNA, so we prefer to study the dissociation rate for this case. 